\title{
Heart rate variability is depressed in the early transitional period for newborns with complex congenital heart disease
}

\author{
Sarah B. Mulkey ${ }^{1,4,5} \cdot$ Rathinaswamy Govindan ${ }^{1} \cdot$ Marina Metzler $^{1} \cdot$ Christopher B. Swisher $^{1} \cdot$ Laura Hitchings $^{1}$. \\ Yunfei Wang ${ }^{2} \cdot$ Robin Baker $^{6,7} \cdot$ G. Larry Maxwell ${ }^{8} \cdot$ Anita Krishnan $^{3,4} \cdot$ Adre J. du Plessis $^{1,4,5}$
}

Received: 5 February 2019 / Accepted: 3 June 2019 / Published online: 25 June 2019

(c) Springer-Verlag GmbH Germany, part of Springer Nature 2019

\begin{abstract}
Purpose To compare early changes in autonomic nervous system (ANS) tone between newborns with complex congenital heart disease (CHD) and newborns without CHD.

Methods We performed a case-control study of heart rate variability (HRV) in newborns with complex CHD [transposition of the great arteries (TGA) or hypoplastic left heart syndrome (HLHS)] and low-risk control newborns without CHD. Cases with CHD were admitted following birth to a pediatric cardiac intensive care unit and had archived continuous ECG data. Control infants were prospectively enrolled at birth. ECG data in cases and controls were analyzed for HRV in the time and frequency domains at $24 \mathrm{~h}$ of age. We analyzed the following HRV metrics: alpha short $\left(\alpha_{\mathrm{S}}\right)$, alpha long $\left(\alpha_{\mathrm{L}}\right)$, root mean square short and long $\left(\mathrm{RMS}_{\mathrm{s}}\right.$ and $\mathrm{RMS}_{\mathrm{L}}$ ), low-frequency (LF) power, normalized LF (nLF), high-frequency (HF) power, and normalized HF (nHF). We used ANOVA to compare HRV metrics between groups and to control for medication exposures. Results HRV data from 57 infants with CHD (TGA, $n=33$ and HLHS, $n=24$ ) and from 29 controls were analyzed. The HRV metrics $\alpha_{\mathrm{S}}, \mathrm{RMS}_{\mathrm{L}}$, LF, and nLF were significantly lower in infants with CHD than in the controls. Due to the effect of normalization, $\mathrm{nHF}$ was higher in CHD infants $(P<0.0001)$, although absolute HF was lower $(P=0.0461)$. After adjusting for medications, $\alpha_{\mathrm{S}}$ and nLF remained lower and nHF higher in newborns with $\mathrm{CHD}(P<0.0005)$.

Conclusions Infants with complex CHD have depressed autonomic balance in the early postnatal period, which may complicate the fetal-neonatal transition.
\end{abstract}

Keywords Congenital heart disease $\cdot$ Autonomic nervous system $\cdot$ Heart rate variability $\cdot$ Newborn

Sarah B. Mulkey

sbmulkey@ childrensnational.org

1 Division of Fetal and Transitional Medicine, Children's National Health System, Washington, DC, USA

2 Biostatistics and Study Methodology, The George Washington University School of Medicine and Health Sciences, Washington, DC, USA

3 Department of Cardiology, Children's National Health System, Washington, DC, USA

4 Department of Pediatrics, The George Washington University School of Medicine and Health Sciences, Washington, DC, USA
5 Department of Neurology, The George Washington University School of Medicine and Health Sciences, Washington, DC, USA

6 Department of Pediatrics, Inova Children's Hospital, Fairfax, VA, USA

Fairfax Neonatal Associates, Fairfax, VA, USA

8 Department of Obstetrics and Gynecology, Inova Fairfax Hospital, Fairfax, VA, USA 


\section{Introduction}

The autonomic nervous system (ANS) plays an essential role in the coordinated control of the cardiovascular and respiratory systems to establish homeostasis during the fetal to neonatal transition. Newborns with complex congenital heart disease (CHD) are at particular risk for hemodynamic instability in the period immediately after birth due to their anatomic cardiovascular anomaly. An additional risk for poor transition in newborns with complex CHD is impaired function of the ANS. Since fetal conditions associated with reduced oxygen and nutrient supply (e.g., complex CHD) may impair maturation of the ANS, the ANS may be unprepared for optimal transition in newborns with complex CHD [1]. Infants with complex CHD who have a poor cardiorespiratory transition can be seen clinically as being at a higher risk for hypotension after birth and requiring greater respiratory support. Furthermore, the autonomic balance in the newborn has not yet established that of the mature ANS, since vagal tone continues to increase through infancy [2].

Hypoplastic left heart syndrome (HLHS) and dextrotransposition of the great arteries (TGA) are two forms of complex CHD that may result in significant hemodynamic instability in the period after birth because of the abnormal circulatory anatomy; during this critical period of adaptation, ANS balance is likely of major importance. Both of these types of CHD are diagnosed in the fetal period or in the immediate postnatal period and require surgery within a few days to weeks after birth. In HLHS, the left side of the heart is small and may provide inadequate systemic and cerebral perfusion; central mixing of oxygenated and deoxygenated blood results in hypoxemic cerebral perfusion. Similarly, in TGA, the aorta and pulmonary arteries are transposed, with the more deoxygenated stream ascending through the aorta into the cerebral circulation. These cardiac anomalies may therefore predispose to both cerebral hypoxemia and hypoperfusion during fetal brain development, leading to potential dysmaturation of the central ANS centers. There is additional risk for ischemic brain injury in the period of transition after birth [3].

ANS function can be measured noninvasively in the infant from the bedside electrocardiogram (ECG) by determining heart rate variability (HRV). HRV is the fluctuation in the length of time between successive heart beats $(R-R$ intervals) and provides a measure of sympathetic and parasympathetic function, and therefore ANS balance $[4,5]$. In the fetus and premature infant, maturation of the ANS toward term gestational age is normally associated with an increase in parasympathetic tone [6], but fetuses with HLHS show reduced HRV compared to healthy control fetuses at a similar gestational age, indicating a delayed maturation of the ANS even prior to birth [7]. However, the ANS balance in infants with complex CHD during the fetal-to-neonatal transition immediately following birth has not been well established. The objective of this study was to compare changes in autonomic tone, as measured by HRV time- and frequency-domain metrics, between infants with complex CHD (TGA or HLHS) and low-risk term control infants. We hypothesized that infants with complex CHD would have lower metrics of HRV (depressed autonomic balance) in the early postnatal preoperative period than the controls.

\section{Methods}

\section{Participants}

We retrospectively studied infants who were diagnosed with complex CHD types TGA or HLHS by echocardiography and underwent surgical repair between August 2012 to April 2016 at Children's National Health System ("Children's National" henceforth) in Washington, DC, and who had available continuous ECG data archived from the cardiac intensive care unit (CICU). Infants with structural brain abnormalities, chromosomal abnormalities, or a birth gestational age of $<36$ weeks were excluded. The study received a waiver of parental consent and Institutional Review Board (IRB) approval at the Children's National Health System.

As part of a prospective study at the Inova Fairfax Hospital (Fairfax, VA, USA), term newborn control infants were prospectively enrolled following informed parental consent and IRB approval at Inova Fairfax Hospital and Children's National between May 2017 and July 2017. Eligible control infants were from uncomplicated pregnancies, labors, and deliveries without significant maternal illness, were $\geq 37$ weeks in gestational age at birth, had a normal birth weight (10th-90th percentile for gestational age), had no postnatal infection, and did not have CHD. The infants did not require intensive care after birth.

\section{Data collection}

The medical history of the infants with CHD (TGA or HLHS) was extracted from the clinical database and included demographic and birth clinical characteristics, need for mechanical ventilator support, and medication exposure. Medications were grouped into four broad classes: sedatives (fentanyl, morphine, midazolam), medications for hypotension (dopamine, epinephrine), neuromuscular blocking agents (vecuronium, rocuronium), and prostaglandin E1 (PGE1). For infants with CHD, continuous ECG recordings from admission to $72 \mathrm{~h}$ of age were retrieved from an institutional Research Data Export archive (IntelliVue Information 
Center, Philips Healthcare, Andover, MA, USA) at a sample rate of $125 \mathrm{~Hz}$. For the controls, we used ECG data collected as part of a combined ECG and high-density electroencephalography study using a Geodesic EEG System 300 (Electrical Geodesics, Inc., Eugene, OR, USA) at a sample rate of $1000 \mathrm{~Hz}$. Clinical and demographic data were collected from study-site medical records. The ECG recording datasets from the controls were approximately $60 \mathrm{~min}$ long and collected at $\leq 48 \mathrm{~h}$ of age. For the CHD group, we selected 60 min of ECG signals at between 23 and $24 \mathrm{~h}$ of age or during the first available 1-h period.

\section{ECG processing and HRV analysis}

ECGs were low-pass filtered with a filter cutoff of $70 \mathrm{~Hz}$ using a Butterworth filter with zero phase distortion. The QRS complex in every cardiac cycle was identified using a recently proposed approach [8], and the beat-to-beat (RRi) interval was calculated. The erroneous (missed/extra) beats were repaired using an established approach [9]. The RRi data were partitioned into 10-min epochs with no overlap. The time-domain metrics were characterized using the detrended fluctuation analysis (DFA) approach [10], and the frequency-domain metrics were characterized using power spectral analysis [11]. For power spectral analysis, the RRis were interpolated using a cubic spline interpolation technique.

To ensure that the difference in ECG sampling rate between CHD infants and controls would not impact results, we systematically downsampled the ECG to 500, 250, 125, and $64 \mathrm{~Hz}$ and calculated the RRi and HRV for each sampling frequency. We compared the HRV metrics obtained for each sampling frequency with those obtained for ECGs sampled at $1 \mathrm{kHz}$. We found no difference between the HRV metrics calculated using 1-kHz-sampled ECGs and the HRV metrics calculated from ECGs sampled at lower sampling frequencies (up to $125 \mathrm{~Hz}$ ) [12]. Thus, the ECGs sampled at $125 \mathrm{~Hz}$ yielded results consistent with those obtained from the ECGs sampled at a higher rate.

\section{Detrended fluctuation analysis: time-domain characterization}

HRV can be measured by time-domain analysis, which evaluates short- and long-term variability in the HR. Long-term variability is influenced by both the sympathetic and parasympathetic nervous systems, whereas short-term variability is influenced more by the parasympathetic nervous system, based on rapid ANS needs [13]. Detrended fluctuation analysis (DFA) is a modified root mean square (RMS) analysis approach that is based on the principles of statistical physics and is designed to counter nonstationarity in RRi [10, 14]. Since accelerations and decelerations in infant heart rate can be caused by infant movement, the heart rate is nonstationary, and DFA provides a way to account for this type of data. This approach is now widely used [15]. Briefly, this method involves (1) calculating the profile function as the cumulative sum of the RRi values; (2) partitioning the profile function into nonoverlapping windows containing a particular number of beats, denoted s; (3) fitting the profile inside each window using a polynomial function; (4) calculating the local fluctuation function in each window as the root mean square of the deviation of the profile from the best fit; and (5) calculating the global fluctuation function as the median of the local fluctuation functions over all windows. Steps 2-5 are repeated for each 10-min epoch. In step (3), we used a fourth-order polynomial function (DFA4), and the value of $s$ was increased in small steps from 6 beats to one-fourth the total number of beats in a 10-min epoch. We derived the following metrics using the fluctuation functions: $\alpha_{\mathrm{S}}$ and $\alpha_{\mathrm{L}}$; these are the slope of the fluctuation function between beats 15 and 30 and the slope of the fluctuation function between beat 35 and the beat corresponding to one-fourth of the total number of samples, respectively (Fig. 1). Similarly, $\mathrm{RMS}_{\mathrm{S}}$ is the maximum of the fluctuation function between beats 15 and 50, whereas $\mathrm{RMS}_{\mathrm{L}}$ is the maximum of the fluctuation function between beats 100 and 150 . The $\alpha$ metrics quantify the autocorrelation in the RRi, whereas the RMS metrics quantify the variability in the RRi. The $\alpha$ metrics are dimensionless quantities. The RMS metrics are in units of seconds

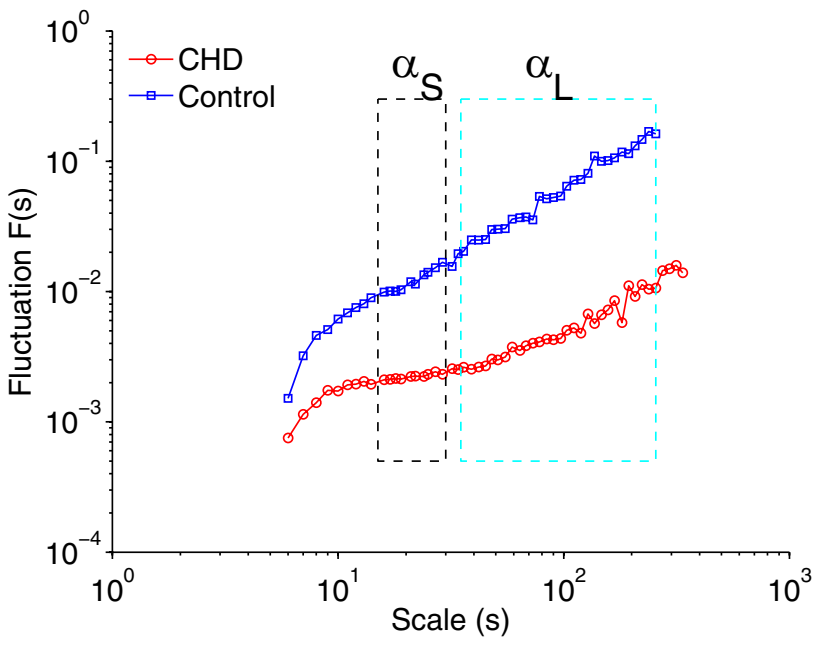

Fig. 1 Sample detrended fluctuation analysis (DFA) plot for an infant with CHD and a control infant. Shown are DFA4 fluctuation functions obtained for $10 \mathrm{~min}$ of RRi of an infant in the control group and of an infant in the CHD group. The fluctuation functions are plotted as a function of the scale $s$ in a log-log representation. 'DFA4' indicates that a fourth-order polynomial was used in the detrending process (refer to text for details). The short-term exponent $\left(\alpha_{\mathrm{S}}\right)$ was calculated from the scale region shown in the black dashed rectangle. The long-term exponent $\left(\alpha_{\mathrm{L}}\right)$ was calculated from the scale region shown in the blue dashed rectangle 
(s). The subscripts ' $S$ ' and ' $L$ ' denote short- and long-term quantities (for details, see [10]).

\section{Spectral analysis}

HRV can also be measured via the frequency domain. Highfrequency (HF) variability reflects parasympathetic function and is influenced by the respiratory rate, while low-frequency (LF) variability is due to a combination of sympathetic and parasympathetic inputs and baroreflex-induced changes in HR [16]. We used Welch's periodogram approach to calculate the power spectrum of the RRi in every 10-min epoch. This method involves partitioning the RRi into 30-s windows (so that the frequency resolution of the resulting spectrum is $0.033 \mathrm{~Hz}$ ) and calculating the periodogram as the square of the magnitude of the Fourier transform of the RRi in that window. We obtained an estimate of the power spectrum as the average of the periodograms from all windows. Using the power spectrum, we calculated the following four metrics: the LF and HF powers as the logarithm of the median spectral power in $0.05-0.25 \mathrm{~Hz}$ and the logarithm of the median spectral power in $0.3-1 \mathrm{~Hz}$, respectively; the normalized LF (nLF) and normalized HF (nHF) as the ratio of the sum of the spectral power in $0.05-0.25 \mathrm{~Hz}$ to the total power and the ratio of the sum of the spectral power in $0.3-1 \mathrm{~Hz}$ to the total power, respectively. Total power was calculated as the sum of the spectral power in $0.05-2 \mathrm{~Hz}$. The normalized spectral powers (nLF and nHF) are dimensionless quantities. The units of the absolute spectral powers are decibels $(\mathrm{dB})$. We did not include the LF/HF ratio since this may not effectively characterize changes in ANS function in all circumstances [17].

\section{Statistical analysis}

The following metrics characterize the sympathetic tone: $\alpha_{\mathrm{s}}, \mathrm{RMS}_{\mathrm{S}}(\mathrm{s})$, and $\mathrm{RMS}_{\mathrm{L}}(\mathrm{s})$, whereas $\mathrm{HF}(\mathrm{dB})$ and $\mathrm{nHF}$ characterize the parasympathetic tone [18]. The metrics LF (dB) and nLF reflect both sympathetic- and parasympathetic-mediated activity. $\alpha_{\mathrm{L}}$ characterizes ultraslow changes in the heart rate, below the frequency of sympathetic tone. All analyses were carried out offline using MATLAB (Mathworks, Inc., MA, USA). The figure was generated with MATLAB. For statistical analysis, the HRVs from all 10-min epochs in 1-h windows were averaged.

Infant clinical characteristics were analyzed using standard measures of central tendency and were compared between TGA and HLHS infants and between CHD infants and controls using the $t$ test and, for categorical variables, the chi-square test. Categorical data were summarized using counts and percentages. Medication use during the data collection period and differences in HRV metrics between the three groups (controls, TGA infants, and HLHS infants) were compared. ANOVA was used to examine differences among the groups, adjusting for the covariates that were significantly different between infants with CHD and controls (medication exposures). The effect of mechanical ventilation on HRV parameters was also assessed. Statistical analysis was performed using SAS 9.3 (SAS Institute Inc., Cary, NC, USA). To deflate the type I error due to multiple comparisons, we considered $P<0.0005$ to indicate a statistically significant difference.

Table 1 Infant demographic and clinical characteristics

\begin{tabular}{|c|c|c|c|c|c|}
\hline Characteristic & TGA $(n=33)$ & HLHS $(n=25)$ & Control $(n=29)$ & $\begin{array}{l}P \text { value TGA vs. } \\
\text { HLHS }\end{array}$ & $\begin{array}{l}P \text { value } \\
\text { CHD vs. } \\
\text { control }\end{array}$ \\
\hline Gestational age, weeks (mean \pm SD) & $38.94 \pm 1.13$ & $38.44 \pm 0.90$ & $39.24 \pm 0.77$ & 0.07 & 0.013 \\
\hline Birth weight, $\mathrm{kg}($ mean $\pm \mathrm{SD})$ & $3.42 \pm 0.42$ & $3.22 \pm 0.52$ & $3.39 \pm 0.30$ & 0.12 & 0.53 \\
\hline Head circumference, $\mathrm{cm}($ mean $\pm \mathrm{SD})$ & $33.87 \pm 1.76$ & $34.10 \pm 1.33$ & $34.83 \pm 1.51$ & 0.58 & 0.019 \\
\hline Male gender $(n, \%)$ & $24(72.7)$ & $17(68)$ & $10(34.5)$ & 0.69 & 0.001 \\
\hline Apgar score at $1 \mathrm{~min}$ (median, range) & $8(4-9)$ & $8(5-9)$ & $8(7-9)$ & 0.0965 & 0.0004 \\
\hline Apgar score at $5 \mathrm{~min}$ (median, range) & $8(7-9)$ & $9(8-9)$ & $9(8-9)$ & 0.0056 & $<0.0001$ \\
\hline Age at study, $\mathrm{h}(\operatorname{mean} \pm \mathrm{SD})$ & $23.3 \pm 8.7$ & $24.7 \pm 6.8$ & $31.0 \pm 13.7$ & 0.4975 & 0.0170 \\
\hline Mechanical ventilation $(n, \%)$ & $19(58)$ & $5(20)$ & NA & $0.004 *$ & NA \\
\hline
\end{tabular}

$P$ values were determined by Student's $t$ test unless noted. The chi-square test was used for male gender

$H L H S$ hypoplastic left heart syndrome, $S D$ standard deviation, $T G A$ transposition of the great arteries 


\section{Results}

\section{Clinical}

Fifty-eight infants with CHD $(\mathrm{TGA}=33$; HLHS $=25)$ were included in the study and compared to 29 control newborns. Demographic and clinical characteristic differences between the infants with CHD and the controls are presented in Table 1. HRV data were analyzed at an earlier mean (SD) hour of age in infants with CHD [23.3(8.7) hours of age in TGA and 24.7(6.8) hours of age in HLHS] compared to controls [31.0(13.7) hours of age] $(P<0.0170)$.

The HRV metrics $\alpha_{\mathrm{S}}, \mathrm{RMS}_{\mathrm{L}}$, LF, and nLF were significantly lower in infants with CHD than in the controls, except nHF, which was higher in the infants with CHD $(P<0.0001)$ (Table 2). Absolute HF was, however, lower in infants with CHD compared to the controls, but this difference did not reach statistical significance $(P=0.0461)$. Mechanical ventilation status did not have an effect on HRV metrics $(P>0.05)$. Due to their medical condition, infants with CHD had multiple medication exposures (Table 3), none of which were present in the controls.
After controlling for the effect of medications, $\alpha_{\mathrm{S}}$ and $\mathrm{nLF}$ remained significantly lower in infants with CHD (TGA and HLHS) compared to the controls, and nHF remained significantly higher $(P<0.0005)$. In addition, $\alpha_{\mathrm{S}}, \mathrm{RMS}_{\mathrm{L}}$, $\mathrm{nLF}$, and nHF all differed between the controls and infants with TGA $(P<0.0005)$, while $\alpha_{\mathrm{S}}$, nLF, and nHF differed between the controls and infants with HLHS $(P<0.0001)$ (Table 2). In summary, the HRV metrics that measure lowfrequency (sympathetic) changes $\left(\alpha_{\mathrm{S}}, \mathrm{RMS}_{\mathrm{L}}, \mathrm{LF}\right.$, and $\left.\mathrm{nLF}\right)$ were lower in the infants with CHD than in the controls.

\section{Discussion}

This study evaluated ANS function in the early transitional preoperative period in newborns with complex CHD by HRV time- and frequency-domain analysis. Compared to controls, we showed that newborns with complex CHD have depression of the sympathetic nervous system and, to a lesser extent, the parasympathetic nervous system during the early postnatal period. Although nHF was higher in infants with CHD than in controls, this is not interpreted as greater changes in parasympathetic tone, since the absolute value of HF was less than in control infants. Due to normalization,

Table 2 Heart rate variability metrics in infants with congenital heart disease and controls

\begin{tabular}{|c|c|c|c|c|c|c|c|c|}
\hline $\begin{array}{l}\text { HRV metric } \\
(\text { mean } \pm S D)\end{array}$ & TGA $(n=33)$ & $\begin{array}{l}\text { HLHS } \\
(n=25)\end{array}$ & $\begin{array}{l}\text { Controls } \\
(n=29)\end{array}$ & $\begin{array}{l}\text { Unadjusted } P \\
\text { value }\end{array}$ & $\begin{array}{l}\text { Control vs } \\
\text { CHD adjusted } \\
P \text { value }{ }^{\#}\end{array}$ & $\begin{array}{l}\text { Control vs } \\
\text { TGA adjusted } \\
P \text { value }\end{array}$ & $\begin{array}{l}\text { Control } \\
\text { vs HLHS } \\
\text { adjusted } P \\
\text { value }^{\#}\end{array}$ & $\begin{array}{l}\text { TGA vs HLHS } \\
\text { adjusted } P \\
\text { value }^{\#}\end{array}$ \\
\hline$\alpha_{\mathrm{S}}$ & $0.59 \pm 0.31$ & $0.64 \pm 0.3$ & $1.4 \pm 0.24$ & $<0.0001^{*}$ & $<0.0001^{*}$ & $<0.0001^{*}$ & $<0.0001^{*}$ & 0.74 \\
\hline$\alpha_{\mathrm{L}}$ & $1.16 \pm 0.23$ & $1.23 \pm 0.23$ & $1.22 \pm 0.19$ & 0.3775 & 0.83 & 0.73 & 0.94 & 0.77 \\
\hline $\mathrm{RMS}_{\mathrm{S}}(\mathrm{s})$ & $0.01 \pm 0.01$ & $0.01 \pm 0.03$ & $0.03 \pm 0.03$ & 0.0014 & 0.26 & 0.14 & 0.51 & 0.43 \\
\hline $\mathrm{RMS}_{\mathrm{L}}(\mathrm{s})$ & $0.04 \pm 0.03$ & $0.05 \pm 0.05$ & $0.13 \pm 0.06$ & $<0.0001^{*}$ & 0.0014 & $0.0004 *$ & 0.01 & 0.5 \\
\hline $\mathrm{nLF}$ & $0.34 \pm 0.15$ & $0.4 \pm 0.13$ & $0.7 \pm 0.1$ & $<0.0001 *$ & $<0.0001^{*}$ & $<0.0001 *$ & $<0.0001^{*}$ & 0.78 \\
\hline $\mathrm{nHF}$ & $0.5 \pm 0.11$ & $0.47 \pm 0.11$ & $0.26 \pm 0.1$ & $<0.0001^{*}$ & $<0.0001^{*}$ & $<0.0001^{*}$ & $<0.0001 *$ & 0.15 \\
\hline $\mathrm{LF}(\mathrm{dB})$ & $-3.77 \pm 0.76$ & $-3.53 \pm 0.81$ & $-2.71 \pm 0.49$ & $<0.0001^{*}$ & 0.42 & 0.13 & 0.91 & 0.11 \\
\hline $\mathrm{HF}(\mathrm{dB})$ & $-4.04 \pm 0.6$ & $-3.95 \pm 0.67$ & $-3.66 \pm 0.56$ & 0.0461 & 0.52 & 0.92 & 0.24 & 0.08 \\
\hline
\end{tabular}

Unadjusted $P$ value from a one-way ANOVA; ${ }^{*}$ indicates an ANOVA adjusted for the medication exposure covariates listed in Table 3; * significant difference between groups

$\alpha_{S}$ alpha short, $\alpha_{L}$ alpha long, $d B$ decibels, $H F$ high frequency, $H L H S$ hypoplastic left heart syndrome, $L F$ low frequency, $n L F$ normalized low frequency, $n H F$ normalized high frequency, $R M S_{S}$ root mean square short, $R M S_{L}$ root mean square long, $T G A$ transposition of the great arteries

Table 3 Medication exposure in infants with congenital heart disease

\begin{tabular}{lllr}
\hline Medication exposure & TGA $(n=33)$ & HLHS $(n=25)$ & $P$ value \\
\hline Neuromuscular blocker agents $(n, \%)$ & $24(73)$ & $7(28)$ & 0.0007 \\
Medications for hypotension $(n, \%)$ & $14(42)$ & $4(16)$ & 0.0312 \\
Sedatives $(n, \%)$ & $26(79)$ & $11(44)$ & 0.0063 \\
PGE $(n, \%)$ & $17(51)$ & $25(100)$ & $<0.0001$ \\
\hline
\end{tabular}

Chi-square test

$H L H S$ hypoplastic left heart syndrome, $T G A$ transposition of the great arteries, $P G E$ prostaglandin E1 
nHF increased because of a relative suppression of sympathetic power (nLF) compared to parasympathetic power, as normalized frequencies are modeled to sum to a value of one. Our study methods employed widely used HRV metrics and newer DFA methods [18-23]. Importantly, the measured autonomic depression remained significant after controlling for medication exposures in the infants with CHD. The knowledge that infants with CHD demonstrate impairment in both sympathetic and parasympathetic balance and have a complex anatomic abnormality that may complicate physiological adaptation during the transitional period may in future be used to improve the care of newborns with complex CHD. For example, impairment in autonomic balance may precede clinically significant hypotension. Since the sympathetic nervous system is important for maintaining cerebral blood flow and cerebral autoregulation, lower autonomic balance could disrupt cerebral blood flow such that it drops below the threshold of autoregulation, affecting cerebral perfusion [24]. With the additional circulatory effects of the anatomic cardiac anomalies in these infants, such an impairment in autonomic balance might further increase the risk of impaired cerebral perfusion and oxygenation during the fetal-to-postnatal transition.

Studies evaluating the normal early maturation of the sympathetic and parasympathetic divisions of the ANS have shown that the sympathetic division begins to develop earlier than the parasympathetic division, which in turn undergoes more accelerated maturation during the third trimester [25, 26]. Adverse intrauterine conditions are known to increase the risk for disturbed fetal development, especially brain development [27-31]. Fetuses with complex CHD, including TGA and HLHS, undergo intrauterine brain development under conditions of tenuous cerebral oxygen delivery. Given the normal rapid development of the central ANS during the third trimester, it is reasonable to speculate that autonomic maturation may also be disturbed in newborns with complex CHD, which is supported by the postnatal HRV data in our study.

To our knowledge, this is the first study of changes in autonomic tone using HRV in infants with CHD during the critical period of fetal-neonatal transition. Several studies have focused on the effect of CHD on HRV characteristics in the fetal period [7, 32]. In a study by Siddiqui et al. [7], using fetal ECG recordings, the authors found a decrease in HRV in fetuses with cyanotic CHD compared to controls. This difference was only significant at gestational ages of between 34 and 38 weeks in fetuses with HLHS, while TGA fetuses showed HRV that was not significantly different from controls at any gestational age [7]. Specific analysis of the sympathetic versus parasympathetic balance was not described [7]. In a study comparing intrapartum fetal heart rate between fetuses with CHD and controls, severe variable decelerations and prolonged decelerations occurred more often in fetuses with CHD than in controls [33]. These features were attributed to a difference in autonomic balance, with a more pronounced parasympathetic response occurring in infants with a potentially lower hypoxemic reserve than in fetuses without CHD. In infants, a hypoxic state may affect ANS function through afferent effects on chemoreceptors that modulate autonomic balance [24].

Unlike Siddiqui's fetal HRV study [7], we found a significant early postnatal depression of autonomic balanceespecially in sympathetic power-in infants with both CHD types, TGA and HLHS. In this regard, our findings are similar to those of Kaltman et al. [20], who found no difference in HRV (in either the LF or the HF power) during the preoperative period between infants with single- versus two-ventricle anatomy [20]. Other studies have described reduced HRV in infants with CHD during the perioperative period [20, 21]. Smith et al. found that during the preoperative period, HLHS infants had reduced HRV compared to age-matched controls [21]; that study excluded infants on mechanical ventilation. Unlike the study by Smith et al. [21], our study focused instead on the very early period after birth. We included infants with TGA and HLHS on mechanical ventilation, and despite a higher number of infants with TGA being on mechanical ventilation, ventilation status did not have an effect on the HRV parameters.

Given the medical acuity of the newborn with complex CHD, we had to consider the effects of other clinical factors, such as medications, on ANS function. Infants undergoing critical care with CHD are exposed to multiple agents with potential autonomic effects, such as the sedatives, medications for hypotension, neuromuscular blocker agents, and prostaglandin E1 that were used in our study population. After controlling for these medication types, we found a persistence of significant autonomic depression in the CHD infants that was shown to be independent of medication exposure.

There are a few studies that examined the relationship between the variability in the RRi and the mean heart rate [35-39]. To mitigate the influence of heart rate on the variability analysis of $\mathrm{RRi}$, it has been suggested that the RRi should be normalized by the mean heart rate or by the exponential of the mean heart rate. Since the exact nature of the relation between the variability in RRi and the mean heart rate is not clear, we have not explored this option in our study. Furthermore, the normalization should not affect the normalized spectral power. Since the results for the normalized low frequency and absolute low frequency show a similar trend, we believe that our results are unbiased characterizations of autonomic tone.

Our study has a number of strengths, but it also has limitations, including those inherent to a retrospective study design. Since our normative dataset for HRV consisted of recordings performed in the first $24-48 \mathrm{~h}$ after birth, it 
enabled us to compare infants with complex CHD to control infants at a similar postnatal age. We were, however, unable to compare the control infants to CHD infants at a later time period since the controls only underwent one ECG session. Infants with CHD were studied at an earlier hour of age than the control infants, due to earlier availability of the CHD infants' data upon admission to our tertiary care children's hospital compared to the control newborns, who were prospectively recruited following birth. While this difference is statistically significant, the biological difference is likely insignificant. Since the ANS maturation after birth is unlikely to be on the scale of hours, we doubt that this small time difference accounts for the differences in ANS maturation between our cases and controls [4, 34]. Furthermore, a larger number of infants with TGA were on mechanical ventilation due to a need for balloon atrial septostomy compared to the infants with HLHS. We controlled for medication exposure and evaluated for the impact of mechanical ventilation in our infants with CHD in order to check if this clinical difference impacted the HRV findings. Children's National is a referral center for neonatal cardiac surgery with no delivery services. Thus, all except one of the CHD cases were outborn, and many were started on PGE1 treatment prior to their arrival at our institution. Prostaglandins are known to exert effects on the ANS and thus might have played some role in the results of our study. The control infants were low-risk term newborns and therefore did not have exposure to postnatal medication or mechanical ventilation, in contrast to the infants with CHD.

\section{Conclusions}

Newborns with complex CHD have depressed HRV metrics of primarily sympathetic balance during the early postnatal transitional period, prior to cardiac surgery, compared to control newborns. Reduced autonomic balance in the early postnatal preoperative period may be due to the impact of prenatal hypoxemia and altered hemodynamics on the developing brain and ANS. Over the last decade, it has become increasingly clear that the high prevalence of long-term neurodevelopmental morbidity in survivors of certain CHD types is likely due to an accumulation of neurologic insults at multiple points in time, including the fetal, pre-, intra-, and postoperative periods. Further studies are required to evaluate the impact of impaired ANS function in newborns with CHD on their clinical course in the cardiac intensive care unit and on long-term neurologic outcome.

\section{Compliance with ethical standards}

Conflict of interest All authors declare that they have no conflict of interest.
Ethical standards This human study was approved by the appropriate ethics committee and has therefore been performed in accordance with the ethical standards laid down in the 1964 Declaration of Helsinki and its later amendments.

\section{References}

1. Petit CJ, Rome JJ, Wernovsky G, Mason SE, Shera DM, Nicolson SC et al (2009) Preoperative brain injury in transposition of the great arteries is associated with oxygenation and time to surgery, not balloon atrial septostomy. Circulation 119(5):709-716

2. Mulkey SB, du Plessis AJ (2019) Autonomic nervous system development and its impact on neuropsychiatric outcome. Pediatr Res 85(2):120-126

3. Mulkey SB, Swearingen CJ, Melguizo MS, Schmitz ML, Ou X, Ramakrishnaiah RH et al (2013) Multi-tiered analysis of brain injury in neonates with congenital heart disease. Pediatr Cardiol 34(8):1772-1784

4. Fyfe KL, Yiallourou SR, Wong FY, Odoi A, Walker AM, Horne RS (2015) The effect of gestational age at birth on post-term maturation of heart rate variability. Sleep 38(10):1635-1644

5. Task Force of the European Society of Cardiology and the North American Society of Pacing and Electrophysiology (1996) Heart rate variability. Standards of measurement, physiological interpretation, and clinical use. Circulation 93(5):1043-1065

6. Fyfe KL, Yiallourou SR, Wong FY, Horne RS (2014) The development of cardiovascular and cerebral vascular control in preterm infants. Sleep Med Rev 18(4):299-310

7. Siddiqui S, Wilpers A, Myers M, Nugent JD, Fifer WP, Williams IA (2015) Autonomic regulation in fetuses with congenital heart disease. Early Hum Dev. 91(3):195-198

8. Kota S, Swisher CB, Al-Shargabi T, Andescavage N, du Plessis A, Govindan RB (2017) Identification of QRS complex in nonstationary electrocardiogram of sick infants. Comput Biol Med 87:211-216

9. Govindan R, Al-Shargabi T, Metzler M, Andescavage NN, Joshi $\mathrm{R}$, du Plessis A (2016) A spike correction approach for variability analysis of heart rate sick infants. Physica A 444:35-42

10. Govindan RB, Wilson JD, Preissl H, Eswaran H, Campbell JQ, Lowery CL (2007) Detrended fluctuation analysis of short datasets: an application to fetal cardiac. Physica D 226(1):23-31

11. Govindan RB, Massaro AN, Niforatos N, du Plessis A (2013) Mitigating the effect of non-stationarity in spectral analysisan application to neonate heart rate analysis. Comput Biol Med 43(12):2001-2006

12. Govindan RB, Massaro A, Kota S, Grabowski R, Wilson JD, du Plessis A (2019) The effect of EKG sampling rate on heart rate variability analysis. In: 41st Annual International Conference of the IEEE Engineering in Medicine and Biology Society (EMBC), Berlin, Germany, 23-27 July 2019 (accepted)

13. Lucchini M, Fifer WP, Sahni R, Signorini MG (2016) Novel heart rate parameters for the assessment of autonomic nervous system function in premature infants. Physiol Meas 37(9):1436-1446

14. Peng CK, Havlin S, Stanley HE, Goldberger AL (1995) Quantification of scaling exponents and crossover phenomena in nonstationary heartbeat time series. Chaos 5(1):82-87

15. Tarvainen MP, Niskanen J-P, Lipponen JA, Ranta-aho PO, Karjalainen PA (eds) (2009) Kubios HRV_a software for advanced heart rate variability analysis. Springer, Heidelberg

16. Malliani A, Lombardi F, Pagani M (1994) Power spectrum analysis of heart rate variability: a tool to explore neural regulatory mechanisms. Br Heart J 71(1):1-2 
17. Billman GE (2013) The LF/HF ratio does not accurately measure cardiac sympatho-vagal balance. Front Physiol 4:26

18. Metzler M, Govindan R, Al-Shargabi T, Vezina G, Andescavage N, Wang Y et al (2017) Pattern of brain injury and depressed heart rate variability in newborns with hypoxic ischemic encephalopathy. Pediatr Res 82(3):438-443

19. Rakow A, Katz-Salamon M, Ericson M, Edner A, Vanpee M (2013) Decreased heart rate variability in children born with low birth weight. Pediatr Res 74(3):339-343

20. Kaltman JR, Hanna BD, Gallagher PR, Gaynor JW, Godinez RI, Tanel RE et al (2006) Heart rate variability following neonatal heart surgery for complex congenital heart disease. Pacing Clin Electrophysiol 29(5):471-478

21. Smith R, Wathen E, Abaci PC, Von Bergen N, Law I, Dick M et al (eds) (2009) Heart rate variability analysis in normal infants and infants with single ventricle anatomy using power spectral density. Comput Cardiol 36:365-368

22. Doheny KK, Palmer C, Browning KN, Jairath P, Liao D, He F et al (2014) Diminished vagal tone is a predictive biomarker of necrotizing enterocolitis-risk in preterm infants. Neurogastroenterol Motil 26(6):832-840

23. Schneebaum Sender N, Govindan RB, Sulemanji M, Al-Shargabi T, Lenin RB, Eksioglu YZ et al (2014) Effects of regional brain injury on the newborn autonomic nervous system. Early Hum Dev 90(12):893-896

24. Mulkey SB, Plessis AD (2018) The critical role of the central autonomic nervous system in fetal-neonatal transition. Semin Pediatr Neurol 28:29-37

25. Longin E, Gerstner T, Schaible T, Lenz T, Konig S (2006) Maturation of the autonomic nervous system: differences in heart rate variability in premature vs. term infants. J Perinat Med 34(4):303-308

26. Patural H, Barthelemy JC, Pichot V, Mazzocchi C, Teyssier G, Damon G et al (2004) Birth prematurity determines prolonged autonomic nervous system immaturity. Clin Auton Res 14(6):391-395

27. Donofrio MT, Massaro AN (2010) Impact of congenital heart disease on brain development and neurodevelopmental outcome. Int J Pediatr 2010:359390. https://doi.org/10.1155/2010/359390

28. Masoller N, Sanz-Cortes M, Crispi F, Gomez O, Bennasar M, Egana-Ugrinovic $\mathrm{G}$ et al (2016) Severity of fetal brain abnormalities in congenital heart disease in relation to the main expected pattern of in utero brain blood supply. Fetal Diagn Ther 39(4):269-278
29. Sun L, Macgowan CK, Sled JG, Yoo SJ, Manlhiot C, Porayette P et al (2015) Reduced fetal cerebral oxygen consumption is associated with smaller brain size in fetuses with congenital heart disease. Circulation 131(15):1313-1323

30. Limperopoulos C, Tworetzky W, McElhinney DB, Newburger JW, Brown DW, Robertson RL Jr et al (2010) Brain volume and metabolism in fetuses with congenital heart disease: evaluation with quantitative magnetic resonance imaging and spectroscopy. Circulation 121(1):26-33 (Epub 2009/12/23)

31. Licht DJ, Shera DM, Clancy RR, Wernovsky G, Montenegro LM, Nicolson SC et al (2009) Brain maturation is delayed in infants with complex congenital heart defects. J Thorac Cardiovasc Surg 137(3):529-536

32. Siddiqui S, Fifer WP, Ordonez-Retamar M, Nugent JD, Williams IA (2017) An antenatal marker of neurodevelopmental outcomes in infants with congenital heart disease. J Perinatol 37(8):953-957

33. Ueda K, Ikeda T, Iwanaga N, Katsuragi S, Yamanaka K, Neki R et al (2009) Intrapartum fetal heart rate monitoring in cases of congenital heart disease. Am J Obstet Gynecol 201(1):64 (e1-e6)

34. Cardoso S, Silva MJ, Guimaraes H (2017) Autonomic nervous system in newborns: a review based on heart rate variability. Childs Nerv Syst 33(7):1053-1063 (Epub 2017/05/16)

35. Zaza A, Lombardi F (2001) Autonomic indexes based on the analysis of heart rate variability: a view from the sinus node. Cardiovasc Res 50(3):434-442 (Epub 2001/05/30)

36. Hayano J, Sakakibara Y, Yamada A, Yamada M, Mukai S, Fujinami $T$ et al (1991) Accuracy of assessment of cardiac vagal tone by heart rate variability in normal subjects. Am J Cardiol 67(2):199-204 (Epub 1991/01/15)

37. Monfredi O, Lyashkov AE, Johnsen AB, Inada S, Schneider H, Wang R et al (2014) Biophysical characterization of the underappreciated and important relationship between heart rate variability and heart rate. Hypertension 64(6):1334-1343 (Epub 2014/09/17)

38. Coumel P, Maison-Blanche P, Catuli D (1994) Heart rate and heart rate variability in normal young adults. J Cardiovasc Electrophysiol 5(11):899-911 (Epub 1994/11/01)

39. Mangin L, Swynghedauw B, Benis A, Thibault N, Lerebours G, Carre F (1998) Relationships between heart rate and heart rate variability: study in conscious rats. J Cardiovasc Pharmacol 32(4):601-607 (Epub 1998/10/22) 